\title{
Mutational analysis of Plasmodium falciparum dihydrofolate reductase and dihydropteroate synthase genes in the interior division of Sabah, Malaysia
}

Tiek Ying Lau ${ }^{1 *}$, Mersumpin Sylvi ${ }^{1}$ and Timothy William ${ }^{2}$

\begin{abstract}
Background: The sulphadoxine/pyrimethamine (SDX/PYR) combination had been chosen to treat uncomplicated falciparum malaria in Malaysia for more than 30 years. Non-silent mutations in dihydrofolate reductase (dhfr) and dihydropteroate synthase (dhps) genes are responsible for the resistance to pyrimethamine and sulphadoxine, respectively. This study reports the mutational analysis of pfdhfr and pfdhps in single Plasmodium falciparum infection isolates from the interior division of Sabah, Malaysian Borneo.

Methods: A total of 22 P. falciparum single infection isolates collected from two districts of the interior division of Sabah from February to November 2010 were recruited for the mutational study of pfdhfr and pfdhps. Both genes were amplified by nested PCR prior to DNA sequencing and mutational analysis.

Results: A total of three pfdhfr and four pfdhps alleles were identified. The most prevalent pfdhfr allele is ANRNL (86\%) involving triple mutation at position 108( $($ to $N), 59(C$ to $R$ ) and 164(I to L). In pfdhps, two novel alleles, SGTGA (73\%) and AAKAA (5\%) were identified. Alleles involving triple mutation in both pfdhfr (ANRNL) and pfdhps (SGTGA), which were absent in Sabah in a study conducted about 15 years ago, are now prevalent.

Conclusions: High prevalence of mutations in SDX/PYR associated drug resistance genes are reported in this study. This mutational study of pfdhps and pfdhfr indicating that SDX/PYR should be discontinued in this region.
\end{abstract}

\section{Background}

Plasmodium falciparum, which causes the most severe form of malaria, is found mainly in the tropical and subtropical regions including Sabah, Malaysian Borneo. The spread of resistance to anti-malarial drugs poses an important health problem. Chloroquine-resistant P. falciparum was first reported in Malaysia in 1966 and the sulphadoxine-pyrimethamine (SDX/PYR) (Fansidar') combination replaced chloroquine in 1979 as first-line treatment for uncomplicated falciparum malaria in Malaysia. The combination of pyrimethamine and sulphadoxine gives a synergistic action against $P$. falciparum by inhibiting the dihydrofolate reductase and dihydropteroate synthase enzymes in the folate biosynthesis pathway,

\footnotetext{
* Correspondence: sophialau@ums.edu.my

'Biotechnology Research Institute, Universiti Malaysia Sabah, Jalan UMS, 88400 Kota Kinabalu, Sabah, Malaysia

Full list of author information is available at the end of the article
}

respectively $[1,2]$. Mutations in the genes encoding for dihydrofolate reductase $(d h f r)$ and dihydropteroate synthase (dhps) are associated with resistance to SDX/ PYR [3].

In Malaysia, artemisinin combination therapy (ACT) is currently being used as the first-line therapy for falciparum malaria. ACT in Sabah consists of either arthemether/lumefantrine or artesunate/mefloquine. This is the first choice for the treatment of uncomplicated $P$. falciparum infections in Sabah according to the State treatment policy guidelines. However, despite this policy, SDX/PYR combination is still occasionally used by field workers to treat $P$. falciparum as presumptive treatment for patients with suspected malaria who live deep in the interior where a timely microscopy result for malaria parasites may not be possible. Besides, SDX/PYR combination is also sometimes used for malaria prophylaxis in this region. 
Molecular techniques based on the detection of mutations in parasite molecules targeted by anti-malarial drugs may offer better tools for monitoring drug resistance. In vitro resistance of $P$. falciparum to PYR is primarily conferred by a non-synonymous point mutation at S108N and is progressively enhanced by mutations at residue N51I and/or C59R of pfdhfr. An additional mutation at $1164 \mathrm{~L}$ is associated with high-level clinical resistance to SDX/PYR $[4,5]$. In pfdhps, point mutations at $\mathrm{A} 437 \mathrm{G}$ and $\mathrm{K} 540 \mathrm{E}$ are considered responsible for SDX resistance. It was thought that the point mutation at 437 is the first event and is associated with a decreased response to SDX [3,6]. Mutations at A581G and A613S in the background of A437G were shown to associate with high clinical resistance to SDX/PYR in South America and Southeast Asia [5,7].

A large-scale study on the clinical efficacy of $P$. falciparum malaria to SDX/PYR in Peninsular Malaysia conducted in mid 1990 s, reported $47 \%$ of the study isolates were resistant to SDX/PYR [8]. A subsequent molecular study reported $87 \%$ of $P$. falciparum had triple mutations in $p f d h f r$ and all isolates having point mutation at codon A437G of pfdhps as indication of drug pressure, accompanied by $81 \%$ point mutation at codon A581G indicating reduced in vitro responsiveness of SDX [4]. The authors speculated that SDX/PYR might be still effective in treating uncomplicated $P$. falciparum malaria in Sabah due to most of the isolates having two or less $d h f r$ mutations. An increasing number of imported cases of malaria as a result of worldwide travel and the use of migrant work forces to and from the neighbouring endemic countries have been reported in Sabah. This makes Sabah vulnerable to the introduction of drug resistant malaria and a reassessment of molecular markers associated with P. falciparum resistance would be timely. In this study, point mutations at pfdhfr and pfdhps genes in P. falciparum isolates from the Interior Division of Sabah, Malaysian Borneo were typed.

\section{Methods}

\section{Study sites and sample collections}

A total of $22 P$. falciparum single infection samples out of 243 total samples collected from the districts of Keningau, and Nabawan in the interior division of Sabah, Malaysian Borneo from February to November in 2010, were analyzed in this study. Ethical clearance was obtained from the Ethical Committee of Ministry of Health Malaysia and the Ethical Committee of Universiti Malaysia Sabah prior Blood samples of approximately $25 \mu \mathrm{l}$ were collected on chromatography paper (Whatman 3MM) and dried before storage in an individual plastic bag for sample collection. Patients' verbal and written consent were obtained before their blood was taken. Sample collection and microscopic examination was carried out by qualified medical laboratory staff at the respective hospitals.

\section{Detection of mutations in pfdhfr and pfdhps}

Genomic DNA was extracted using QIAmp ${ }^{\circ}$ DNA Mini Kit (QIAGEN, Hilden, Germany), according to the manufacturer's recommendation. Plasmodium species were identified by nested-PCR using genus and speciesspecific primers based on the small subunit ribosomal RNA gene (ssrRNA). Single P.falciparum infections were recruited in this study.

pfdhfr was amplified in nested PCR according to previously described method with minor modifications [9]. Primary round amplification comprised of $4 \mu \mathrm{l}$ DNA template, $0.25 \mu \mathrm{M}$ each primers, $1.5 \mathrm{mM} \mathrm{MgCl}_{2}, 200 \mu \mathrm{M}$ dNTPs, $1 \times$ PCR buffer, and $2.5 \mathrm{U}$ of Taq in a $20 \mu \mathrm{l}$ reactions. Cycling conditions were performed as follow; $94^{\circ} \mathrm{C}$ for $3 \mathrm{~min}$, followed by 45 cycles of $94^{\circ} \mathrm{C}$ for $30 \mathrm{sec}, 45^{\circ} \mathrm{C}$ for $45 \mathrm{sec}$, and $72^{\circ} \mathrm{C}$ for $45 \mathrm{sec}$ and finally $72^{\circ} \mathrm{C}$ for $5 \mathrm{~min}$. Nested PCR was performed with $2 \mu \mathrm{l}$ DNA template from the first round PCR product, $0.25 \mu \mathrm{M}$ primers, $1.5 \mathrm{mM}$ $\mathrm{MgCl}_{2}, 200 \mu \mathrm{M}$ dNTPs, $1 \times$ PCR buffer, and $1 \mathrm{U}$ of Taq in a $20 \mu \mathrm{l}$ reactions. The PCR was carried at $94^{\circ} \mathrm{C}$ for $3 \mathrm{~min}$, followed by 40 cycles at $94^{\circ} \mathrm{C}$ for $1 \mathrm{~min}, 45^{\circ} \mathrm{C}$ for $1 \mathrm{~min}$, $72^{\circ} \mathrm{C}$ for $1 \mathrm{~min}$ and finally $72^{\circ} \mathrm{C}$ for $10 \mathrm{~min}$.

Amplification of pfdhps was performed in nested PCR according to previously described method with minor modifications [10]. First round of PCR was carried out in a $20 \mu \mathrm{l}$ reaction containing $4 \mu \mathrm{l}$ template, $0.25 \mu \mathrm{M}$ primers, $2.5 \mathrm{mM} \mathrm{MgCl} 2,200 \mu \mathrm{M}$ dNTPs, $1 \times$ PCR buffer, and $2.5 \mathrm{U}$ of Taq at the following PCR conditions, $94^{\circ} \mathrm{C}$ for $5 \mathrm{~min}$, followed by 30 cycles $94^{\circ} \mathrm{C}$ for $55 \mathrm{sec}, 45^{\circ} \mathrm{C}$ for $45 \mathrm{sec}, 72^{\circ} \mathrm{C}$ for $45 \mathrm{sec}$ and finally $72^{\circ} \mathrm{C}$ for $5 \mathrm{~min}$. Nested PCR comprising $2 \mu \mathrm{l}$ DNA template from the first round PCR product, $0.25 \mu \mathrm{M}$ primers, $1.5 \mathrm{mM} \mathrm{MgCl}_{2}$, $200 \mu \mathrm{M}$ dNTPs, $1 \times$ PCR buffer, and $1.0 \mathrm{U}$ of Taq in a $20 \mu \mathrm{l}$ reaction. PCR was carried out at $94^{\circ} \mathrm{C}$ for $3 \mathrm{~min}$, followed by 40 cycles at $94^{\circ} \mathrm{C}$ for $1 \mathrm{~min}, 45^{\circ} \mathrm{C}$ for $1 \mathrm{~min}$, $72^{\circ} \mathrm{C}$ for $1 \mathrm{~min}$ and $72^{\circ} \mathrm{C}$ for $10 \mathrm{~min}$. Primer sequences for nested PCR amplification of pfdhps and pfdhfr is shown in Table 1.

PCR products were purified using QIAquick Gel Extraction Kit (QIAGEN, Hilden, Germany) prior to DNA sequencing. Detection of the mutations was performed using the MEGA version 5 through comparative analysis with the wild type sequence (GenBank accession numbers are XM_001351443 and Z30654 for pfdhfr and pfdhps, respectively). Single nucleotide polymorphisms were verified through the bidirectional sequencing.

\section{Results}

\section{Mutational analysis of pfdhfr}

Based on the analysis of 5-codon alleles in pfdhfr, the wild type allele, ANCSI was absent in the $22 P$. falciparum 
Table 1 Primer sequence for the amplification of pfdhfr and pfdhps

\begin{tabular}{|c|c|c|c|c|}
\hline \multicolumn{2}{|c|}{ PCR reaction } & \multirow{2}{*}{$\begin{array}{l}\text { Primer pair sequence } \\
\text { Pfdhfr_D1 5' TTAATATTTCTCCTTTTA 3' }\end{array}$} & \multirow{2}{*}{$\begin{array}{l}\text { Expected size } \\
718 \mathrm{bp}\end{array}$} & \multirow{2}{*}{$\begin{array}{l}\text { Reference } \\
{[9]}\end{array}$} \\
\hline pfdhfr & Nest 1 & & & \\
\hline & & Pfdhfr_D2 5' CATTTTATTATTCGTTTCT 3' & & \\
\hline & Nest 2 & Pfdhfr_M1 5' TTTATGATGGAACAAGTCTGC 3' & $648 \mathrm{bp}$ & \\
\hline & & Pfdhfr_M5 5' AGTATATACATCGCTAACAGA 3' & & \\
\hline \multirow[t]{4}{*}{ pfdhps } & Nest 1 & Pfdhps_N185 5' TGATACCCGAATATAAGCATAATG 3' & $1031 \mathrm{bp}$ & [10] \\
\hline & & Pfdhps_N218 5' ATAATAGCTGTAGGAAGCAATTG 3' & & \\
\hline & Nest 2 & Pfdhps_Rc 5'GGTATTITTGTTGAACCTAAACG 3' & $728 \mathrm{bp}$ & \\
\hline & & Pfdhps_Rd 5'ATCCAATTGTGTGATTTGTCCAC 3' & & \\
\hline
\end{tabular}

isolates in the interior division of Sabah. Three different alleles, namely ANRNL, ANRNI and AIRNI were detected (Table 2a). The most prevalent allele in this study is ANRNL (86.4\%), which was absent in previous study conducted in Sabah about 15 years ago [4]. All isolates had mutation at position S108N and C59R accompanied by $86.4 \%(n=19)$ mutation at I164L. Approximately 9.1\% $(n=2)$ of the total isolates showing pfdhfr double mutations at position S108N and C59R alone (ANRNI). One isolate from this region had additional mutation at N51I, which has never been reported in Sabah, giving rise to allele AIRNI. This allele was prevalent in P. falciparum isolates from Kelantan, Peninsular Malaysia where clinical resistance to SDX/PYR has been reported in various locations [4].

\section{Mutational analysis of pfdhps}

Four alleles of pfdhps namely SGTGA, SGKGA, SGKAA and AAKAA but not the wild type allele SAKAA, were identified in this study (Table 2b). SGTGA is the most prevalent allele which present in $72.7 \%(\mathrm{n}=16)$ of the total study isolates. Only $13.6 \%(\mathrm{n}=3)$ of the P. falciparum

Table 2 Dihydrofolate reductase (dhfr) and dihydropteroate synthase (dhps) alleles in Plasmodium falciparum isolates from the interior division of Sabah, Malaysian Borneo

\begin{tabular}{|c|c|c|c|c|c|c|}
\hline \multirow[t]{2}{*}{ (a) } & \multicolumn{5}{|c|}{ pfdhfr amino acid position } & \multirow{2}{*}{$\begin{array}{l}\text { Prevalence (\%) } \\
n=22\end{array}$} \\
\hline & 16 & 51 & 59 & 108 & 164 & \\
\hline & A & N & $R$ & N & L & 86.4 \\
\hline & A & 1 & $R$ & N & 1 & 4.5 \\
\hline & A & $\mathrm{N}$ & $\mathrm{R}$ & $\mathrm{N}$ & । & 9.1 \\
\hline \multirow[t]{6}{*}{ (b) } & \multicolumn{5}{|c|}{ pfdhps amino acid position } & Prevalence (\%) \\
\hline & 436 & 437 & 540 & 581 & 613 & $n=22$ \\
\hline & $\mathrm{S}$ & G & $T$ & G & A & 72.7 \\
\hline & $A$ & A & K & A & A & 4.5 \\
\hline & $\mathrm{S}$ & G & K & A & A & 9.1 \\
\hline & $S$ & G & K & G & A & 13.6 \\
\hline
\end{tabular}

*Single letter amino acid denoting the wild type and point mutations are indicated with italics. The wild type allele of pfdhfr is ANCSI and pfdhps is SAKAA. isolates had the SGKGA allele, which was previously the most common allele (76\%) in Sabah [4]. A novel point mutation at position 540 from lysine (K) to threonine (T), instead of glutamic acid (E) was prevalent in Sabah (72.7\%). K540T point mutation is unique to $P$. falciparum isolates in Sabah. It is noteworthy that $\mathrm{K} 540 \mathrm{E}$ was reported in Sabah in the previous study but was absent in this study. Besides, one isolate had mutation at S436A, which is also novel to Sabah P. falciparum isolates.

\section{Discussion}

Single or multiple mutations at residues A16V, N51I, C59R, S108N and I164L of pfdhfr have been reported to be associated with resistance to antifolate drugs in falciparum malaria treatment. Of these, mutations at N51I, C59R, S108N and I164L were shown to correlate with resistance to pyrimethamine $[5,11]$. Mutation at A16V was specifically associated with cycloguanil resistance [12]. Mutation at S108N was thought to be the first event which resulted in reduce in vitro responsiveness to pyrimethamine, followed by point mutation at N51I and/ or C59R and finally at position I164L. A previous report implicated that an accumulation of mutations were associated with increasing resistance to pyrimethamine [11]. In this study, $86.4 \%$ of the $P$. falciparum isolates had triple mutations at C59R, S108N and I164L (ACNRNL). In accordance with the previous study, all of the isolates showed mutation $\mathrm{S} 108 \mathrm{~N}$ as expected in populations where SDX/PYR has been used extensively. However, in contrast to the previous study that showed $2 \%$ prevalence of wild type allele at codon 59 [5], there was no wild type allele at this position in the current study.

Triple mutations involving the residue I164L were commonly found in areas with high levels of clinical resistance to SDX/PYR [5]. It is worth noting that even though mutation at $\mathrm{A} 16 \mathrm{~V}$ was not detected in the present study, the presence of triple mutation at C59R, S108N and I164L could provide cross-resistance to cycloguanil $[13,14]$. Cycloguanil is the active metabolite of proguanil, an antifolate drug that is commonly used in the prophylaxis of $P$. falciparum malaria. Prophylactic 
use of proguanil was widespread in Malaya in the early 1950 s and it is used in combination with atovaquone as prophylaxis by travellers to Malaysia.

A previous study showed that double mutation at C59R and $\mathrm{S} 108 \mathrm{~N}$ was prevalent in Sabah and predicted not to cause clinical resistance to pyrimethamine [4]. Recently, studies conducted in Angola and Iran also reported that double mutation at residues $\mathrm{C} 59 \mathrm{R}$ and $\mathrm{S} 108 \mathrm{~N}$ are more likely to act as a predictor for the development of resistance to antifolate drugs $[15,16]$. Taking this into consideration, additional mutation at residue I164L found in this study at high prevalence could be the pivotal mutation that causes clinical resistance of P. falciparum to SDX/ PYR therapy in Sabah. Previous report showed that the presence of three $d h f r$ point mutations were correlated with clinical resistance to SDX/PYR, regardless of dhps alleles [4].

In pfdhps, mutation at position A437G is associated with sulphonamide resistance, while additional point mutations at S436A, K540E, A581G and A613S might increase the degree of resistance. Plasmodium falciparum, harbouring double mutations at residues A437G and K540E (SGEAA), were known to be associated with resistance to sulphadoxine $[17,18]$. In Sabah, allele SGKGA was predominant ten years ago, while the current finding showed only approximately $14 \%(3 / 22)$ allele SGKGA. Additional mutation at K540T that gave rise to allele SGTGA was the most prevalent allele (72.7\%). Mutation at K540T is unique to the $P$. falciparum isolates from Sabah, as amino acid change from lysine to glutamic acid (K540E) was commonly found. High prevalence of K540T in $P$. falciparum isolates in this region due to the nonsynonymous amino acid substitution (AAA $\rightarrow$ ACA) instead of K540E (AAA $\rightarrow$ GAA) need to be highlighted. Plasmodium falciparum isolates carrying $540 \mathrm{~T}$ have replaced isolates with $540 \mathrm{E}$ within the timeframe of a decade. High prevalence of $540 \mathrm{~T}$ and absence of $540 \mathrm{E}$ indicating that this codon has high tendency subjecting to natural selection and might enhance the drug resistance pressure of $P$. falciparum carrying this mutation. Besides, recent studies reported a change from lysine to asparagine $(\mathrm{K} 540 \mathrm{~N})$ at a few geographical locations, indicating mutations at codon 540 are more likely to change to different amino acids $[19,20]$. In this study, mutation at K540T occurred as a result of non-synonymous mutation at nucleotides coding for lysine (AAA) to threonine (ACA). Triple mutations at residues S436A, K540T and A581G (SGTGA) could contribute to the resistance to sulphadoxine in Sabah, thus decreasing the efficacy of SDX/PYR. One novel allele (AAKAA) involving mutation at residue S436A was found in one of the isolates and is not thought to cause resistance to sulphadoxine. This allele is sometime referred as wild type allele in a previous report [21].
Table 3 Allele combinations of pfdhfr and pfdhps in Plasmodium falciparum isolates from the interior division of Sabah, Malaysian Borneo

\begin{tabular}{ll}
\hline Pfdhfr-pfdhps allele combinations & Prevalence (\%) \\
\hline ANRNL-SGTGA & 72.7 \\
ANRNL-SGKGA & 13.6 \\
AIRNI-AAKAA & 4.5 \\
ANRNI-SGKAA & 9.1 \\
\hline
\end{tabular}

* Single letter amino acid denoting the wild type and point mutations are indicated with italics.

A four-allele combination of pfdhfr-pfdhps, namely ANRNI-SGKAA, AIRNI-AAKAA, ANRNL-SGKGA and ANRNL-SGTGA were formed in this study (Table 3). The sextuple mutations (ANRNL-SGTGA), which comprise triple mutations in both genes, are most prevalent (72.7\%) in Sabah. Overall, the present study reported a progressive mutation in $d h f r$ and dhps of $P$. falciparum isolates in the interior division of Sabah, where triple mutations are now prevalent in both genes compared to the previous study with double mutations on both genes. A sextuple allele combination of pfdhfr-pfdhps is associated with high-grade clinical resistance to SDX/PYR, which was found mainly in Africa [22,23]. The other three alleles found in Sabah range from triple mutation to quintuple mutations (ANRNI-SGKAA, AIRNI-AAKAA, ANRNL-SGKGA), which could modulate varying degree of resistance to SDX/PYR therapy.

\section{Conclusions}

This preliminary study showed that mutations in $p f d h f r$ and pfdhps, which are associated with SDX/PYR resistance are now prevalent in Sabah. The spread of resistant $P$. falciparum to SDX/PYR in Sabah making this drug combination is no longer effective in combating $P$. falciparum in this geographical region. This report also confirms that treatment with this combination is unlikely to be effective and should thus be discontinued. Artemisinin-based combination therapy (artesunate/ mefloquine or artemether/lumefantrine) should continue to be used as the preferred treatment for falciparum malaria. Further study is required to elucidate the close relationship of these mutations with the susceptibility of P. falciparum isolates to SDX/PYR in Sabah.

\section{Competing interests}

The authors declare that they have no competing interests.

\section{Authors' contributions}

TYL conceived and designed the study. WT was responsible for coordinating sample collection. MS performed the experiments and data analysis. TYL MS and WT participated in manuscript preparation. All authors read and approved the final manuscript.

\section{Acknowledgements}

We are grateful to the hospital directors and laboratory staff of the respective hospitals for their assistance in sample collection. We are thankful 
to the Ministry of Health Malaysia and Sabah Health Department for approving this study to be conducted and to the Malaria Research Centre for providing the Plasmodium positive controls. This study was funded by the Ministry of Higher Education under Fundamental Research Grant Scheme (FRG0276-SG-2/2011).

\section{Author details}

${ }^{1}$ Biotechnology Research Institute, Universiti Malaysia Sabah, Jalan UMS, 88400 Kota Kinabalu, Sabah, Malaysia. ${ }^{2}$ Infectious Disease Department, Queen Elizabeth Hospital, Karung Berkunci No. 2029, Jalan Penampang, 88560 Kota Kinabalu, Sabah, Malaysia.

Received: 19 August 2013 Accepted: 4 December 2013

Published: 10 December 2013

\section{References}

1. Chuley JD, Watkins WM, Sixsmith DG: Synergistic antimalarial activity of pyrimethamine and sulfadoxine against Plasmodium falciparum in vitro. Am J Trop Med Hyg 1984, 33:325-330.

2. Ferone R: Folate metabolism in malaria. Bull World Health Organ 1977, 55:291-298

3. Wang P, Lee CS, Bayoumi R, Djimde A, Doumbo O, Swedberg G, Dao LD, Mshinda H, Tanner M, Watkins WM, Sims PF, Hyde JE: Resistance to antifolates in Plasmodium falciparum monitored by sequence analysis of dihydropteroate synthetase and dihydrofolate reductase alleles in a large number of field samples of diverse origins. Mol Biochem Parasitol 1997, 89:161-177.

4. Cox-Singh J, Zakaria R, Abdullah MS, Rahman HA, Nagappan S, Singh B: Differences in dihydrofolate reductase but not dihydropteroate synthase alleles in Plasmodium falciparum isolates from geographically distinct areas in Malaysia. Am J Trop Med Hyg 2001, 64:28-31.

5. Plowe CV, Cortese JF, Djimde A, Nwanyanwu OC, Watkins WM, Winstanley PA, Estrada-Franco JG, Mollinedo RE, Avila JC, Cespedes JL, Carter D, Doumbo OK: Mutations in Plasmodium falciparum dihydrofolate reductase and dihydropteroate synthase and epidemiologic patterns of pyrimethamine-sulfadoxine use and resistance. J Infect Dis 1997, 176:1590-1596.

6. Diourte Y, Djimde A, Doumbo OK, Sagara I, Coulibaly Y, Dicko A, Diallo M, Diakité M, Cortese JF, Plowe CV: Pyrimethamine-sulfadoxine efficacy and selection for mutations in Plasmodium falciparum dihydrofolate reductase and dihydropteroate synthase in Mali. Am J Trop Med Hyg 1999, 60:475-478.

7. Biswas S, Escalante A, Chaiyaroj S, Angkasekwinai P, Lal AA: Prevalence of point mutations in the dihydrofolate reductase and dihydropteroate synthetase genes of Plasmodium falciparum isolates from India and Thailand: a molecular epidemiologic study. Trop Med Int Health 2000, 5:737-743.

8. Lokman Hakim S, Sharifah Roohi SW, Zurkurnai Y, Noor Rain A, Mansor SM, Palmer K, Navaratnam V, Mak JW: Plasmodium falciparum: increased proportion of severe resistance (RII and RIII) to chloroquine and high rate of resistance to sulfadoxine-pyrimethamine in Peninsular Malaysia after two decades. Trans R Soc Trop Med Hyg 1996, 90:294-297.

9. Duraisingh MT, Curtis J, Warhurst DC: Plasmodium falciparum: Detection of polymorphisms in the dihydrofolate reductase and dihydropteroate synthetase genes by PCR and restriction digestion. Exp Parasitol 1998, 89:1-8.

10. Wang P, Brooks DR, Sims PFG, Hyde JE: A mutation-specific PCR system to detect sequence variation in the dihydropteroate synthetase gene of Plasmodium falciparum. Mol Biochem Parasitol 1995, 71:115-125.

11. Cowman AF, Morry MJ, Biggs BA, Cross GA, Foote SJ: Amino acid changes linked to pyrimethamine resistance in the dihydrofolate reductasethymidylate synthase gene of Plasmodium falciparum. Proc Natl Acad SCi US A 1988, 85:9109-9113.

12. Sirawaraporn W, Sathitkul T, Sirawaraporn R, Yuthavong Y, Santi DV: Antifolate-resistant mutants of Plasmodium falciparum dihydrofolate reductase. Proc Natl Acad Sci U S A 1997, 94:1124-1129.

13. Peterson DS, Milhous WK, Wellems TE: Molecular basis of differential resistance to cycloguanil and pyrimethamine in Plasmodium falciparum malaria. Proc Natl Acad Sci U S A 1990, 87:3018-3022.

14. Foote SJ, Galatis D, Cowman AF: Amino acids in the dihydrofolate reductase-thymidylate synthase gene of Plasmodium falciparum involved in cycloguanil resistance differ from those involved in pyrimethamine resistance. Proc Natl Acad Sci U S A 1990, 87:3014-3017.

15. Fortes F, Dimbu R, Figueiredo P, Neto Z, Do Rosário VE, Lopes D: Evaluation of prevalence's of pfdhfr and pfdhps mutations in Angola. Malar J 2011, 10:22

16. Zakeri S, Farahani MS, Afsharpad M, Salehi M, Raeisi A, Djadid ND: High prevalence of the $437 \mathrm{G}$ mutation associated with sulfadoxine resistance among Plasmodium falciparum clinical isolates from Iran, three years after the introduction of sulfadoxine-pyrimethamine. Int J Infect Dis 2010, 14:123-128.

17. Bwijo B, Kaneko A, Takechi M, Zungu IL, Moriyama Y, Lum JK, Tsukahara T, Mita T, Takahashi N, Bergqvist Y, Björkman A, Kobayakawa T: High prevalence of quintuple mutant $d h p s / d h f r$ genes in Plasmodium falciparum infections seven years after introduction of sulfadoxine and pyrimethamine as first line treatment in Malawi. Acta Trop 2003, 85:363-373

18. Nzila AM, Mberu EK, Sulo J, Dayo H, Winstanley PA, Sibley $\mathrm{CH}$, Watkins WM: Towards an understanding of the mechanism of pyrimethamine sulfadoxine resistance in Plasmodium falciparum: genotyping of dihydrofolate reductase and dihydropteroate synthase of Kenyan parasites. Antimicrob Agents Chemother 2000, 44:991-996.

19. Lumb V, Sharma YD: Novel K540N Mutation in Plasmodium falciparum dihydropteroate synthetase confers a lower level of sulfa drug resistance than does a K540E mutation. Antimicrob Agents Chemother 2011, $55: 2481-2482$

20. Vinayak S, Alam MT, Mixson-Hayden T, McCollum AM, Sem R, Shah NK, Lim P, Muth S, Rogers WO, Fandeur T, Barnwell JW, Escalante AA, Wongsrichanalai C, Ariey F, Meshnick SR, Udhayakumar V: Origin and evolution of sulphadoxine resistant Plasmodium falciparum. PLoS Pathog 2010, 6:1000830.

21. Enosse S, Magnussen P, Abacassamo F, Gómez-Olivé X, Rønn AM, Thompson R, Alifrangis M: Rapid increase of Plasmodium falciparum dhfr/ dhps resistant haplotypes, after the adoption of sulphadoxinepyrimethamine as first line treatment in 2002, in southern Mozambique. Malar J 2008, 7:115.

22. Karema C, Imwong M, Fanello Cl, Stepniewska K, Uwimana A, Nakeesathit S, Dondorp A, Day NP, White NJ: Molecular correlates of high-level antifolate resistance in Rwandan children with Plasmodium falciparum malaria. Antimicrob Agents Chemother 2010, 54:477-483.

23. Zeile I, Gahutu JB, Shyirambere C, Steininger C, Musemakweri A, Sebahungu F, Karema C, Harms G, Eggelte TA, Mockenhaupt FP: Molecular markers of Plasmodium falciparum drug resistance in southern highland Rwanda. Acta Trop 2012, 12:50-54.

doi:10.1186/1475-2875-12-445

Cite this article as: Lau et al:: Mutational analysis of Plasmodium falciparum dihydrofolate reductase and dihydropteroate synthase genes in the interior division of Sabah, Malaysia. Malaria Journal 2013 12:445.

\section{Submit your next manuscript to BioMed Central and take full advantage of:}

- Convenient online submission

- Thorough peer review

- No space constraints or color figure charges

- Immediate publication on acceptance

- Inclusion in PubMed, CAS, Scopus and Google Scholar

- Research which is freely available for redistribution 